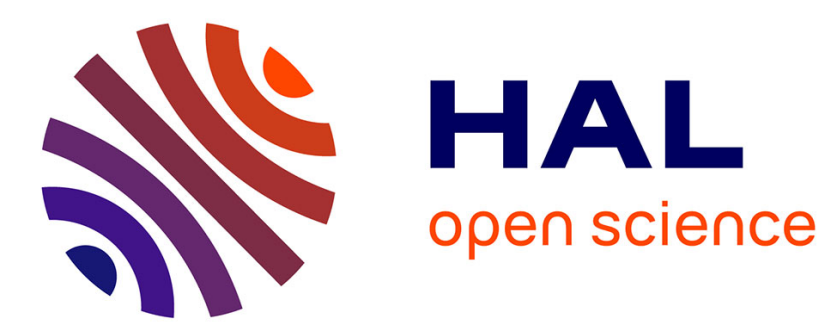

\title{
Elastic Metasurfaces for Deep and Robust Subwavelength Focusing and Imaging
}

Yabin Jin, Wan Wang, Abdelkrim Khelif, Bahram Djafari-Rouhani

\section{To cite this version:}

Yabin Jin, Wan Wang, Abdelkrim Khelif, Bahram Djafari-Rouhani. Elastic Metasurfaces for Deep and Robust Subwavelength Focusing and Imaging. Physical Review Applied, 2021, 15, pp.024005 (10). 10.1103/PhysRevApplied.15.024005 . hal-03414227

\section{HAL Id: hal-03414227 \\ https://hal.science/hal-03414227}

Submitted on 4 Nov 2021

HAL is a multi-disciplinary open access archive for the deposit and dissemination of scientific research documents, whether they are published or not. The documents may come from teaching and research institutions in France or abroad, or from public or private research centers.
L'archive ouverte pluridisciplinaire HAL, est destinée au dépôt et à la diffusion de documents scientifiques de niveau recherche, publiés ou non, émanant des établissements d'enseignement et de recherche français ou étrangers, des laboratoires publics ou privés. 


\title{
Elastic metasurfaces for deep and robust subwavelength focusing
}

\section{and imaging}

\author{
Yabin Jin ${ }^{1, *}$, Wan Wang ${ }^{1}$, Abdelkrim Khelif ${ }^{2}$, Bahram Djafari-Rouhani ${ }^{3}$ \\ ${ }^{1}$ School of Aerospace Engineering and Applied Mechanics, Tongji University, 200092 Shanghai, China \\ ${ }^{2}$ Institut FEMTO-ST, CNRS, Université de Bourgogne Franche-Comté 15B Avenue des Montboucons, \\ CEDEX, F-25030 Besançon, France \\ ${ }^{3}$ Institut d'Electronique, de Microélectonique et de Nanotechnologie, UMR CNRS 8520, Département de \\ Physique, Université de Lille, 59650 Villeneuve d'Ascq, France
}

*Corresponding author: 083623jinyabin@tongji.edu.cn

\begin{abstract}
Metasurfaces are planar metamaterials with a flat surface and a subwavelength thickness that are able to shape arbitrary wavefronts such as focusing or imaging. There is a broad interest in the literature about subwavelength focusing/imaging based on bulk metamaterials while the utilization of metasurfaces for elastic waves has rarely been reported. Here, we present a new type of elastic metasurface consisting of a line of gradient resonant pillars for robust deep subwavelength focusing and imaging of elastic waves in a plate. Numerical approaches supported by analytic Huygens-Fresnel demonstrations show that the subwavelength full width at half maximum (FWHM) behaves linearly as a function of the ratio $F / D$ where $F$ is the measured focal length and $D$ the metasurface length. We discuss the range of $F / D$ where FWHM remains smaller than half a wavelength in the near field. The focal length $F$ and the FWHM exhibit stable performances when submitted to disorder perturbations in the geometrical parameters and to frequency fluctuations. We show that the enhanced focusing resolution with smaller FWHM can be very beneficial for energy harvesting since the output electric power can be increased by more than one order of magnitude. The proposed elastic metasurfaces bring a new way for high resolution focusing and imaging which is useful for applications in various domains such as energy harvesting, wave sensing, communication, nondestructive evaluation.
\end{abstract}




\section{Introduction}

A metasurface is an array of subwavelength thickness units with nonhomogeneous wave response that defines a new principle for wave control[1-3], in particular by introducing a gradient in the phase or amplitude of the reflection and transmission coefficients of neighboring units. The wave response of the metasurface can be tailored by designing properly the geometry of the units to realize diverse abnormal applications. In recent years, this concept has been widely developed for optical[4-6] and acoustical [7-11] waves and extended to elastic waves [12-18] and mechanical systems, especially owing to the significant role of elastic and mechanical waves as information carrier for communication[19, 20]. Comparing to phononic crystals and acoustic metamaterials[21-25], acoustic metasurfaces have the advantage of a compact size that promote the design of thin and lightweight structures for different applications such as wave focusing, imaging, absorption, isolation, modal conversion, among others.

The focusing resolution of acoustic/elastic waves in the far-field suffers from diffraction limit due to the loss of evanescent waves which carry subwavelength information and decay exponentially from the source/object. This is referred to as Abbé or Rayleigh criterion. It is often mentioned in most references that the full width at half maximum (FWHM) of a focusing transverse intensity field is of the order of $\lambda / 2$ ( $\lambda$ is wavelength) due to the diffraction limit. Different mechanisms are proposed to break the diffraction limit so that a smaller FWHM than $\lambda / 2$ can be achieved to improve the focusing resolution. The development of phononic crystals and acoustic metamaterials provides negative effective refractive index[26-31] by dispersion analysis and homogenization scheme to overcome the diffraction limit by collecting or magnifying the evanescent waves. Super-oscillation acoustic wave packets can be generated and further superimposed to a diffraction-limit-broken spot by designing acoustic meta-lenses which originates from the Schrodinger equation in quantum mechanical system[32, 33]. An alternative approach is based on the utilization of time reversal acoustics [34, 35]. In this approach, regardless of the complexity of the scattering media, a set of waves are created by a distribution of transducers that precisely retraces all of the complex paths of the scattered field and synchronously converges to the original source. Recently, a gradient index acoustic metasurface is exhibited to achieve focusing spots beyond the diffraction limit via spoof surface acoustic wave modulation[36]. Farfield imaging and edge detection of subwavelength objects have also been proposed by designing 
61 a resonator array to amplify spatial frequencies and a binary phase grating to convert 62 subwavelength components to propagating mode[37]. Focusing of spherical waves beyond the diffraction limit is demonstrated with anti-causal Green's function based on membrance-type acoustic metasurface[38]. One can notice that these realizations are for air-borne sound waves; therefore, their principles to beat the diffraction limit cannot be directly applied to mechanical waves where multiple polarizations and a coupling between the vibrational components are involved.

In this work, we propose a new type of pillared elastic metasurface for the purpose of subwavelength focusing and imaging whose FWHM is much smaller than half a wavelength. It consists of a line of resonant pillars with gradient phase response whose diameters are subwavelength. It is found that the transmitted wave can be regarded as a sum of the incident wave and the scattered waves from resonant pillars. Each resonant pillar plays as a secondary emitter source to generate point-like scattering waves[14]. Then, several transmitted waves with a gradient phase shift fully covering a $2 \pi$ range can be realized by designing an array of gradient resonant pillars as shown in Sec.2. In Sec.3 focusing phenomena are achieved whose FWHM of the transverse intensity profile is significantly less than half a wavelength. This is based on the physical mechanism of interference and diffraction between the gradient sub-sources in the near field region. The subwavelength focusing of the designed metasurface is numerically demonstrated by using the finite element method and the trend of FWHM as a function of the focal distance $F$ and the metasurface length $D$ is investigated. The numerical results are theoretically supported on the basis of the Huygens-Fresnel principle applied in the frame of a Green's function method. In Sec.4 it is further demonstrated that the subwavelength focusing is robust against disorders in the geometrical parameters or frequency change of the incident wave, which would be beneficial for the practical fabrication and measurement issues. We show that the decrease in the FWHMs of focusing spots makes the pillared metasurfaces of significant interest for the purpose of energy harvesting. Indeed, they allow an enhancement by more than one order of magnitude of the output electric power that can be recovered by piezoelectric materials placed at the location of the spots. In Sec.5, we also demonstrated the subwavelength imaging effect by the proposed metasurface with FWHM less than half a wavelength. We finally summarize the work in Sec.6. 


\section{Metasurface units}

For a line of identical pillars on a plate, there are two main low frequency resonances, namely compressional and bending modes. The resonant frequencies are directly related to the geometrical parameters of the pillars, such as height and diameter[14]. We set the initial geometric parameters as follows: pillar's diameter $d=120 \mu \mathrm{m}$, pillar's height $h=239 \mu \mathrm{m}$, plate's thickness $e=145 \mu \mathrm{m}$, period of the array $a=150 \mu \mathrm{m}$. Cubic silicon is chosen for the entire solid structure unless specifically mentioned. The choice of material is not a key factor for the observed trends and other common materials such as aluminum for machining process and steel or polymer for 3D printer can be used as well. Also, the results can be produced at different frequency scales by changing all the geometrical parameters by the same scale factor. The above geometrical parameters are chosen appropriately such that the second bending and first compressional modes are superposed at 8.05MHz. This choice is beneficial in order to keep a high level of transmission while allowing a span of the phase shift over $2 \pi$ when changing gradually the height of the pillars.

The object is to design a line of gradient pillars (e.g. gradient in pillar's height) to steer incident wavefronts into desired forms. In Fig.1a, a plane wave focusing effect is illustrated with a pillared metasurface. The resonant properties of one pillar in the metasurface are obtained by considering a periodical structure of period $a=150 \mu \mathrm{m}$ with this pillar and applying the periodic boundary condition based on Bloch theorem. The fundamental antisymmetric Lamb wave (flexural wave with main component along $z$ axis perpendicular to the plate, called $A_{0}$ ) is excited and propagated along $y$ axis to interact with the pillars and the vibrating information about the resonant frequencies and transmission coefficients are obtained by the finite element method. To be mentioned that Fig.1a is not the whole space of simulation in our design, for instance, the surrounding perfect matched layers around the sample are not shown.

For the pillar's parameters mentioned above, the second bending and first compressional modes are superposed at $8.05 \mathrm{MHz}$. The amplitude and phase of the out-of-plane displacement component $u_{z}$ in the downstream on the plate with respect to the reference (same detected point on the plate without pillars) are respectively shown in Fig.1b by sweeping the pillar's height in the unit cell from $100 \mu \mathrm{m}$ to $400 \mu \mathrm{m}$ while keeping their diameter $d$ and the period $a$ constant for the sake of simplicity. It is found that the transmitted phase spans a range from $-\pi$ to $\pi$ as revealed by the black curve while the transmitted amplitude keeps a relatively high level as shown by the level 
of color in Fig. 1b. The average value of the squares of the transmission coefficient where phase covers $2 \pi$ is 0.79 , which supports an overall sufficient energy in the transmitted field. It should also be noted that the structure made of one line of pillars can possibly convert part of the incident $\mathrm{A}_{0}$ mode into $\mathrm{S}_{0}$ mode; however, we have checked that this conversion remains weak, so that the transmitted wave is still dominated by $\mathrm{A}_{0}$ mode. Therefore, the phase and amplitude in Fig.1b keep the same no matter the detected position in the downstream of the pillars' line, namely very close to the pillars (e.g. until half a wavelength) or in the far field. This is in contrast to the case of a single-sided pillared phononic crystal plate (a set of pillars deposited on one side of the plate in the propagation direction), where the incident A0 mode wave is able to generate a strong coexistence of $\mathrm{A}_{0}$ and $\mathrm{S}_{0}$ modes, as shown in $\operatorname{Ref[39].}$

(a)

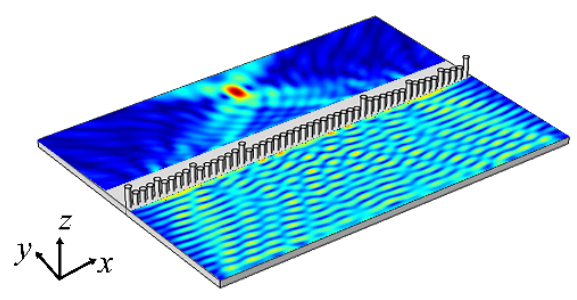

(b)

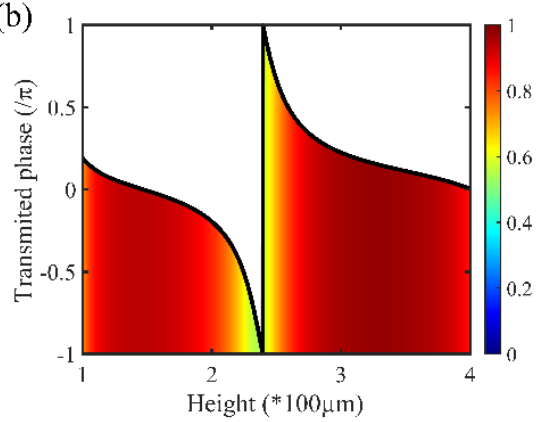

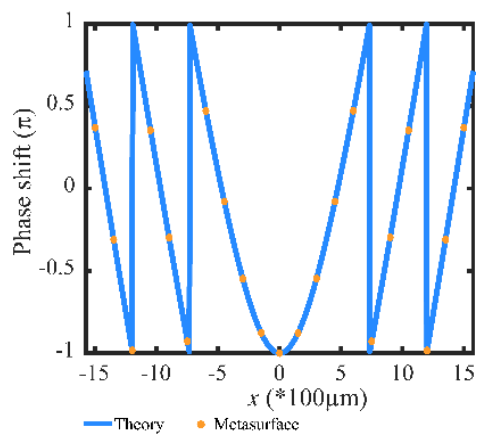

Figure 1. (a) Illustration of the gradient pillared metasurface for focusing effect; (b) the variation response of transmitted phase (black curve) and amplitude (color level) in a periodic array as a function of the pillar's height $h$ in the unit cell. (c) the theoretical phase profile (blue line) for $\mathrm{F}=\lambda$ and $\Phi(x=0)=-\pi$ from Eq.(1) and the discrete phases of the 21 selected pillars for the metasurface (yellow dots).

Taking advantage of a full $2 \pi$ phase span and sufficient high transmission amplitudes, we can design a gradient phase meta-line of pillars to realize anomalous transmitted wavefronts based on the generalized Snell's law. To be noted, the thickness of this metasurface, i.e. the diameter of the pillars, is less than $1 / 3$ of the incident wavelength, namely $\lambda=423 \mu \mathrm{m}$ for the $\mathrm{A}_{0}$ mode at 8.05 MHz. In our metasurface approach, the bearing capacity and rigidity of the plate are conserved, showing an excellent candidate in compact solid devices at micro or macro scales. As an example, a gradient metasurface can be designed for plane wave focusing as shown in Fig. 1a. Different from a metasurface unit with plate's tailoring $[12,13]$, the resonant pillars will directly make impact on the wave focusing while keeping the rigidity of the plate. 


\section{Sub-wavelength focusing}

With full tunability of the transmitted phase, a metasurface consisting of different units can be designed for desired wavefront functions based on the generalized Snell's law. The aim here is to design a focusing effect with an incident plane wave. Therefore, the phase profile $\Phi(x)$ along the $x$ direction can be easily derived as

$$
\Phi(x)=\frac{2 \pi}{\lambda}\left(\sqrt{F^{2}+x^{2}}-F\right)+\Phi(x=0)
$$

where $\lambda=423 \mu \mathrm{m}$ is the wavelength of the $\mathrm{A}_{0}$ Lamb mode at $8.05 \mathrm{MHz}$, and $F$ is the focal length.

First, 21 pillars are considered along $x$ for the metasurface with an interval of $150 \mu \mathrm{m}$, about $1 / 3$ of the wavelength, and the focal length $F$ is fixed to $\lambda=423 \mu \mathrm{m}$. Also, the phase of the central pillar $(\mathrm{x}=0)$ is set to $-\pi$ corresponding to the strongest resonant status. The discrete required phase for the other pillars (yellow dots in Fig.1c) can be easily calculated by Eq. (1) and the corresponding heights can be further obtained from the relationship (black line) in Fig. 1b. The transmitted intensity field of the focusing effect by the pillared metasurface is shown in Fig.2a. The measured focal length is found to be $1.06 \lambda$ and the FWHM (obtained from the transverse intensity along the $x$ axis crossing the spot) reaches a subwavelength value of $0.37 \lambda$ (Fig. 2c). To validate the subwavelength focusing effect, we adopt the Huygens-Fresnel principle with a calculation based on the Green's function method (Fig. 2b).

The Huygens-Fresnel principle would consist of superposing the emitted fields of the pillars while assuming that each pillar is a point source emitting with the amplitude $A_{i}$ and phase $\varphi_{i}$ as defined from Fig. 1b and c. Such a calculation can be analytically performed using a Green's function approach, namely the displacement field response is given by

$$
w_{i}\left(r_{i}\right)=A_{i} W\left(r_{i}\right) e^{-i \varphi_{i}}
$$

Here $W(r)$ is the Green function associated with the equation of motion of flexural wave in the plate and satisfies the following equation

$$
\left(\nabla^{4}-k^{4}\right) W(r)=\delta(r)
$$

where $\nabla^{4}$ is the biharmonic operator, $k$ is the wavenumber. The solution can be found in the form [40] 
where $H_{0}$ and $K_{0}$ are the zeroth-order Hankel function and modified Bessel function of the second kind, respectively. In order to obtain results closer to the simulated realistic metasurface, we adopt the wavenumber obtained from simulation to include in Eq.(4), then apply the Huygens-Fresnel principle. The calculated focusing field is displayed in Fig.2b whose measured $F$ is $0.92 \lambda$ and the FWHM is $0.32 \lambda$ as shown in Fig. $2 \mathrm{c}$ and $d$.
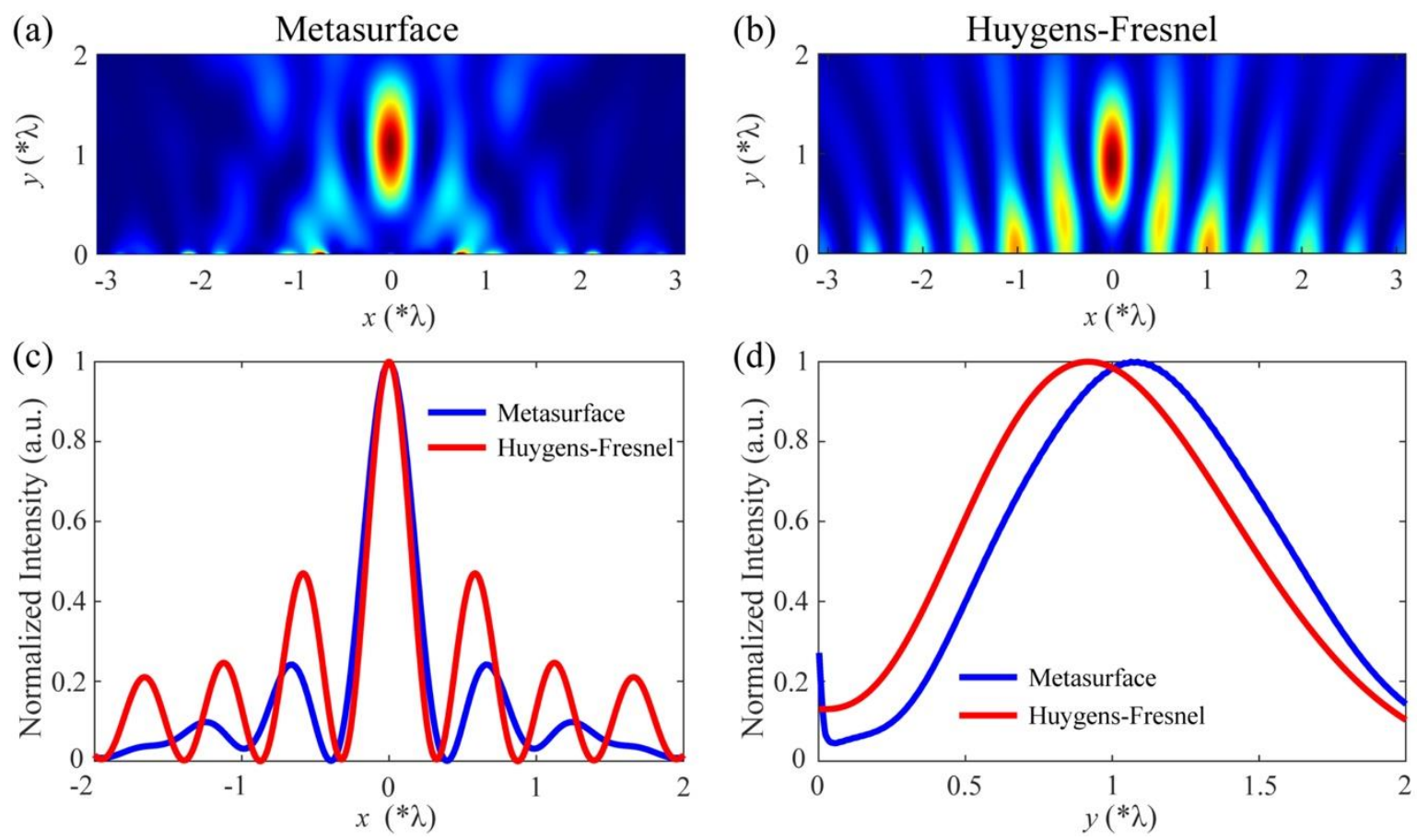

Figure 2. Intensity field of plane wave focusing effect by (a) pillared metasurface, (b) analytically calculated Huygens-Fresnel principle with the Green's function approach. (c) the intensity along the $x$ axis crossing the focusing spot. (d) the intensity along the $y$ axis crossing the focusing point.

The subwavelength focusing is simultaneously dependent upon the focal length $F$ and the metasurface length $D$. The length $\mathrm{D}$ is defined along the $x$ axis and directly depends on the number of the pillars. We study this dependence for several design values of $F$, namely $\lambda, 3 \lambda$ and $5 \lambda$. For each $F$ value, we sweep the metasurface length $D$ and calculate the intensity field distributions and the corresponding FWHM. These calculations are performed either numerically for the actual metasurface (called M1, M2, M3) or in the frame of the Huygens-Fresnel principle based on the Green's function approach (called G1, G2, G3). For both methods, we find in general a linear relationship between the FWHM and the ratio $F / D$ as shown by the circles and star dots in Fig.3. 
To be noted that in the ratio $F / D$ we use the actual measured focal length $F$ rather than the nominal one. We define a fitting function $\mathrm{FWHM}=a^{*} F / D+b$ for the two approaches as shown by the solid and dashed lines with the fitting coefficient $a, b$ shown in Table 1. For the metasurface simulation approach, the circles above $F / D=0.3$ (corresponding to FWHM over $0.5 \lambda$ ) are excluded from the fitting process because for a low number of pillars (or a small D), the focusing quality is lost and the results deviate too much from the extrapolated straight line. For a similar reason, we disregard in the G1 case the upper limit star where $F / D=0.254$ corresponding to $\mathrm{FWHM}=0.389$.

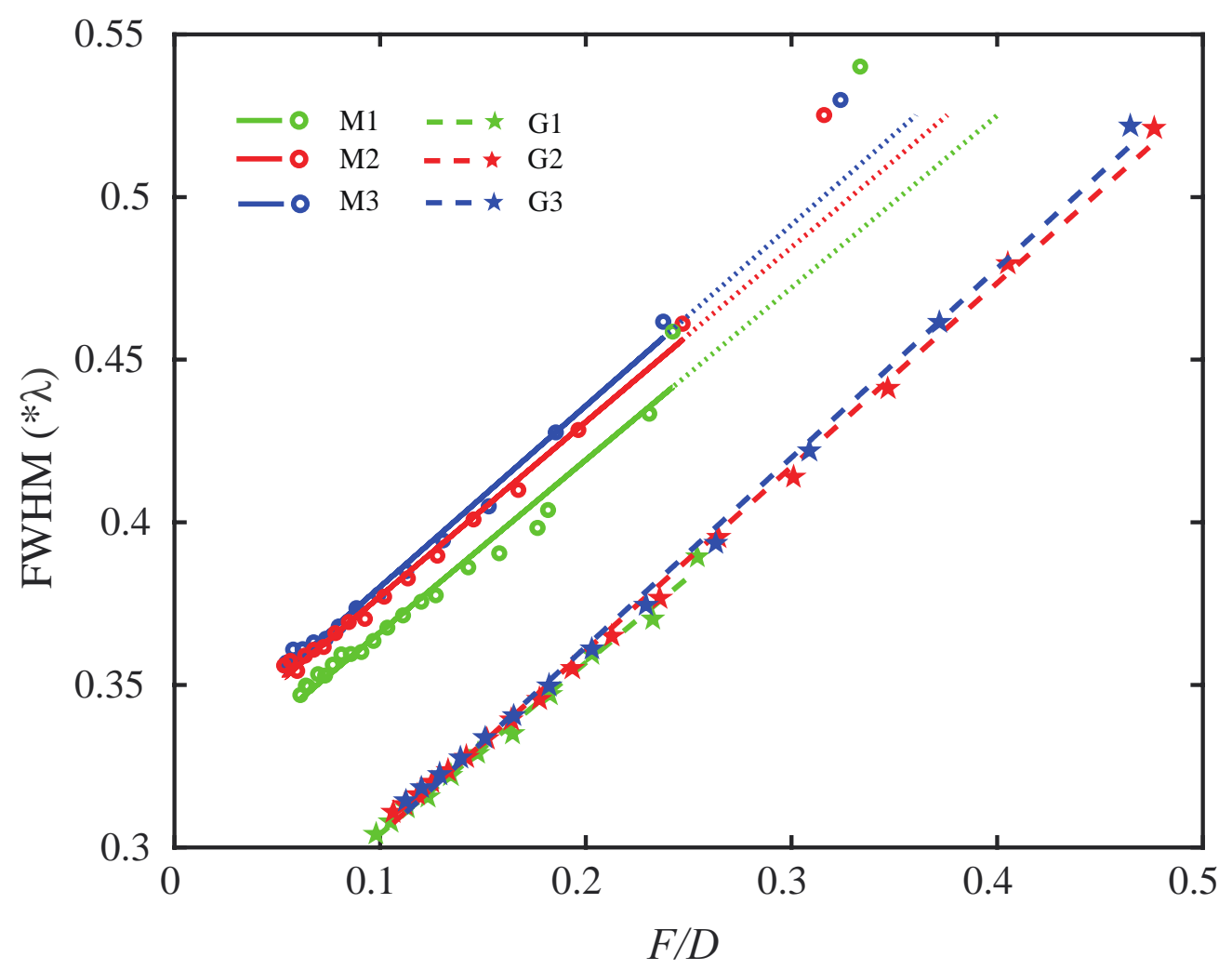

Figure 3. The relation between the $F / D$ and FWHM by the metasurface simulation (M1, M2 and M3 stand for designed $F$ as $\lambda, 3 \lambda, 5 \lambda$ ), and the Green's function based Huygens-Fresnel (G1, G2 and G3 stand for designed $\mathrm{F}$ as $\lambda, 3 \lambda, 5 \lambda$ ) approaches with their corresponding fitting curves (solid lines for $\mathrm{M}$ and dotted lines for $\mathrm{G}$ ) in the form of $\mathrm{FWHM}=a^{*} F / D+b$. To be noted that in the ratio $F / D$ we use the measured focal length $F$.

Table. 1 Fitting coefficient

\begin{tabular}{lll}
\hline Case & $a$ & $b$ \\
\hline M1 & 0.530 & 0.313 \\
M2 & 0.536 & 0.324 \\
M3 & 0.557 & 0.325
\end{tabular}




\begin{tabular}{lll} 
G1 & 0.527 & 0.252 \\
G2 & 0.565 & 0.247 \\
G3 & 0.582 & 0.245 \\
\hline
\end{tabular}

For the Green's function based Huygens-Fresnel approach, each pillar is assumed as a point source which is defined by a delta function force. The gradient sources are realized by setting the amplitude and phase shown in Fig.1. Generally, the slopes of the fitting curves by the two approaches are similar for different cases. However, the Huygens-Fresnel approach do not take into consideration the coupling effect among the pillars in the metasurface, which explains the mismatch in fitting coefficient $b$ and the different configurations of side lobes as shown in Fig.2a and $b$.

From Fig.3, one can observe a broad parameter's space for realizing subwavelength focusing with FWHM less than $0.5 \lambda$ which the physical mechanism is the interference and diffraction of the gradient sub-sources in the near field region[41, 42] with the near field length defined as $L_{\mathrm{N}}=$ $D^{2} / 4 \lambda$. When the ratio $F / D$ goes above 0.3 and 0.4 , the FWHM becomes larger than $0.5 \lambda$ for the simulation and analytical methods, respectively. For different designed focal lengths, the linear relationships remain valid and keep stable with both approaches, showing a guidance for the design of subwavelength focusing metasurfaces with proper $F / D$.

As long as the ratio $F / D$ keeps at a low level in the near field, the subwavelength focusing effect (FWHM less than $0.5 \lambda$ ) can be maintained. To give an illustration, we further propose a metasurface consisting of 255 pillars for a designed focal length of $5 \lambda$ and show the focusing information in Fig.4, where a focusing spot is clearly observed in Fig.4a. The measured FWHM and focal length are $0.34 \lambda$ and $4.8 \lambda$ as can be seen from Fig. $4 \mathrm{~b}$ and c, respectively, corresponding to $F / D=0.079$. 

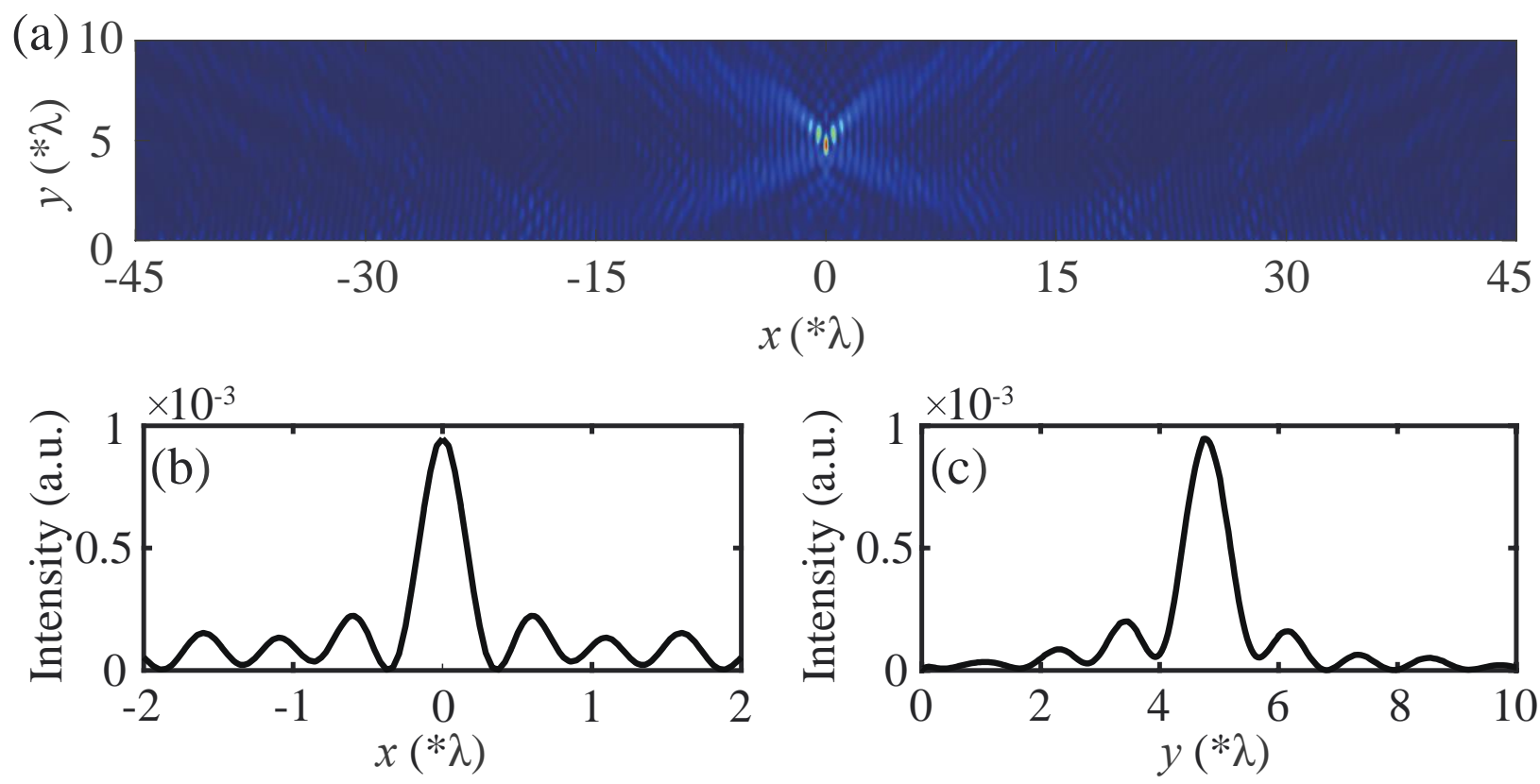

Figure 4. (a) The intensity field of plane wave focusing effect from pillared metasurface with 255 pillars for a designed focal length of $5 \lambda$. (b) the intensity along the $\mathrm{x}$ axis crossing the focusing spot showing a FWHM of $0.34 \lambda$ (c) the intensity along the y axis crossing the focusing point showing a focal length of $F=4.8 \lambda$.

\section{Robustness of subwavelength focusing and application to energy harvesting}

In this section we first demonstrate the robustness of sub-wavelength features against disorder in the geometrical parameters of the metasurface or a shift in the precise frequency of the incident wave. Since the focusing properties of the proposed pillared metasurface depend on the geometry and not the choice of the constituent material, we employ in this section a plate made of a very common material such as aluminum and propose a design of the metasurface in the millimeter scale.

Following the same principle as in Sec.2, the geometrical parameters of the proposed aluminum metasurface consisting of 51 pillars are chosen as follows: plate's thickness $e=10 \mathrm{~mm}$, width of unit cell $7.5 \mathrm{~mm}$, pillar's radius $3 \mathrm{~mm}$. The pillar's height varies from $5.7 \mathrm{~mm}$ to $18 \mathrm{~mm}$ to fully cover $2 \pi$ phase shift for a working frequency of $99 \mathrm{kHz}$ corresponding to a wavelength of $23.8 \mathrm{~mm}$. The ratio between the metasurface's thickness and the wavelength is only $1 / 4$. The designed focal length is chosen as $\lambda$ and the phase of the central pillar is chosen as $\Phi(x=0)=-\pi$. We consider a disorder value in position, radius and height for all the pillars simultaneously in the metasurface as it can appear in practical fabrications. The disorder degree is quantified as $\gamma$, meaning that the introduced disorder value is randomly chosen between 0 and $\gamma \mathrm{mm}$. A maximum 
disorder degree $\gamma=0.5 \mathrm{~mm}$ is considered which corresponds to a maximum error of $16.7 \%$ for pillar's radius, much higher than most error levels in practical machining process fabrication.

In Fig.5a, we plot the measured focal length and FWHM as a function of the disorder parameter $\gamma$. Each disorder case is sampled 5 times. For each disorder level, the vertical bars stand for the obtained maximum and minimum values and the solid line stands for the average values over the 5 times sampling. Without disorder $(\gamma=0)$, the focal length and the FWHM of the focusing spot are $1.04 \lambda$ and $0.32 \lambda$, respectively. It can be noticed that the curves are almost horizontal which clearly supports the strong robustness of subwavelength features against such disorders that can happen in fabrications and experiments. Although the metasurface is designed for single frequency at $99 \mathrm{kHz}$, we evaluate the subwavelength focusing with a frequency shift since experimentally the exciting wave packet is broadened over a certain frequency range. In Fig.5b, we consider a frequency bandwidth ratio (bandwidth divided by the central frequency) about $12 \%$, with a frequency range from $93 \mathrm{kHz}$ to $105 \mathrm{kHz}$. The position of the focal length has a small variation around the designed value of $\lambda$. On the other hand, the FWHM of the focusing spot almost keeps flat below $0.5 \lambda$, with a high robustness. The analysis of robustness helps to ensure the validity of the subwavelength focusing in real sample fabrication and experimental measurements.
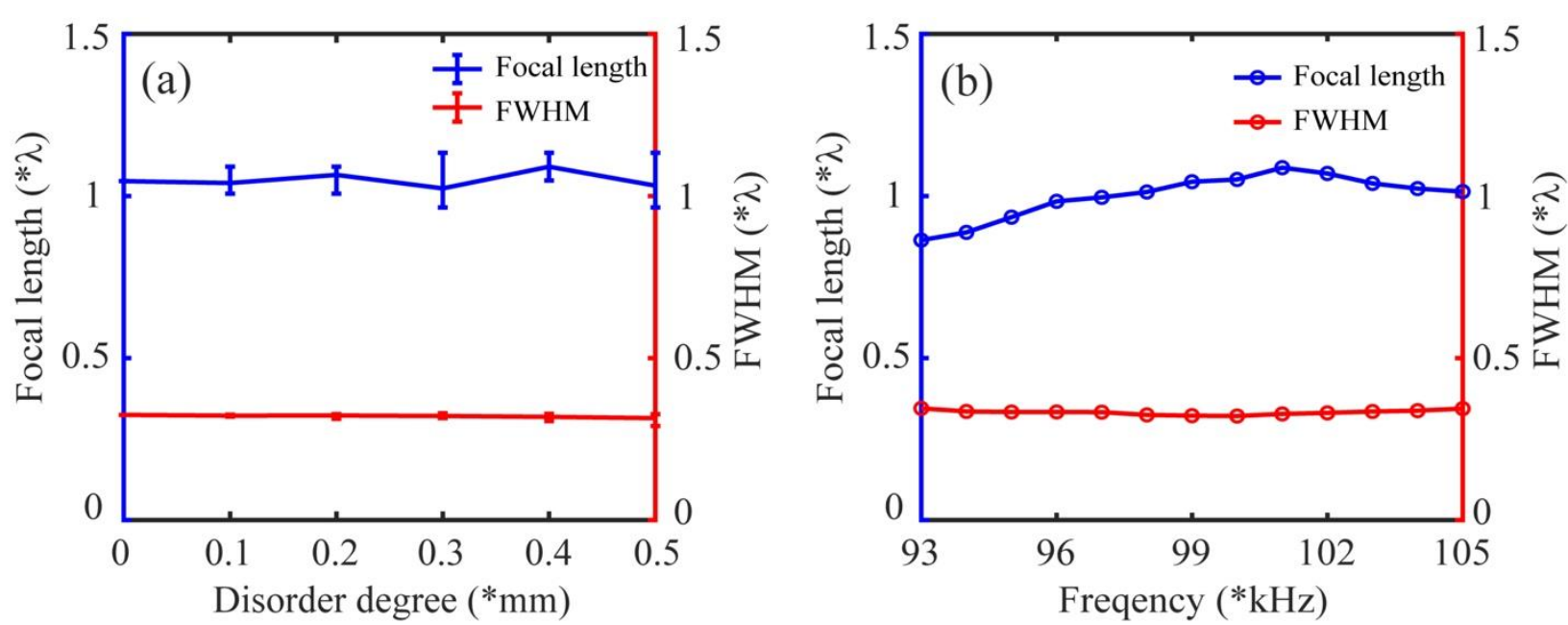

Figure 5. Robustness of subwavelength focusing against disorder effects. (a) we show the evolutions of the focal length and the FWHM of the focusing spots as a function of the disorder degree. (b) the measured focal length and the FWHM of subwavelength focusing against frequency shifts

Besides improving the resolution in non-destructive detection, a reduction in the FWHM focusing will increase the confinement of elastic energy that can be used for energy harvesting. 
276 We design a PZT thin layer attached to the opposite side of the plate located at the red dotted 277 rectangle in the inset of Fig.6. For the AC electrical circuit, the output power $P$ can be derived 278 as[43]

$$
P=\frac{1}{2} I^{2} R=\frac{R}{2}\left(\frac{\omega|\eta|}{1+\omega \varepsilon_{33} R L_{1} L_{2} / t}\right)^{2} \quad, \quad \eta=-\frac{e}{2} \int_{A}\left(e_{31} \frac{\partial^{2} w}{\partial x^{2}}+e_{32} \frac{\partial^{2} w}{\partial y^{2}}\right) d A
$$

where $I$ is the current, $R$ the resistance chosen as $1200 \Omega, e_{31}=e_{32}=-6.62281 \mathrm{Cm}^{-2}$ the piezoelectric constants of PZT, $\varepsilon_{33}=1433.6 \varepsilon_{0}$ the dielectric permittivity with $\varepsilon_{0}$ being the vacuum permittivity, $L_{1}=13.1 \mathrm{~mm}$ and $L_{2}=47.55 \mathrm{~mm}$ the sizes of the red dotted rectangle along $x$ and $y$, $e=10 \mathrm{~mm}$ the plate's thickness, $t=1 \mathrm{~mm}$ the thickness of PZT layer. Once the geometry and the material properties of the piezoelectric layer are fixed, the main factor affecting the output power is the curvature of the plate's surface along $x$ and $y$ in the red dotted rectangle. These curvatures appear in the integral defining the parameter $\eta$ - in Eq. (5). We adopt the focusing field from the Green's function approach (as in Fig.2b) and calculate the output power for different FWHM values of G1 case in Fig.3. The results for the output power are presented as the dotted-line in Fig.6. One can notice that the power is 16 times higher at $\mathrm{FWHM}=0.287 \lambda$ than at $F W H M=0.318 \lambda$. Since the elastic strain energy is more confined for higher focusing resolution situations, the surface's curvatures will be also enhanced, resulting in higher output electric powers. Therefore, the subwavelength focusing performance of the elastic metasurfaces brings a significant impact on the increase of the energy density for harvesting application. 


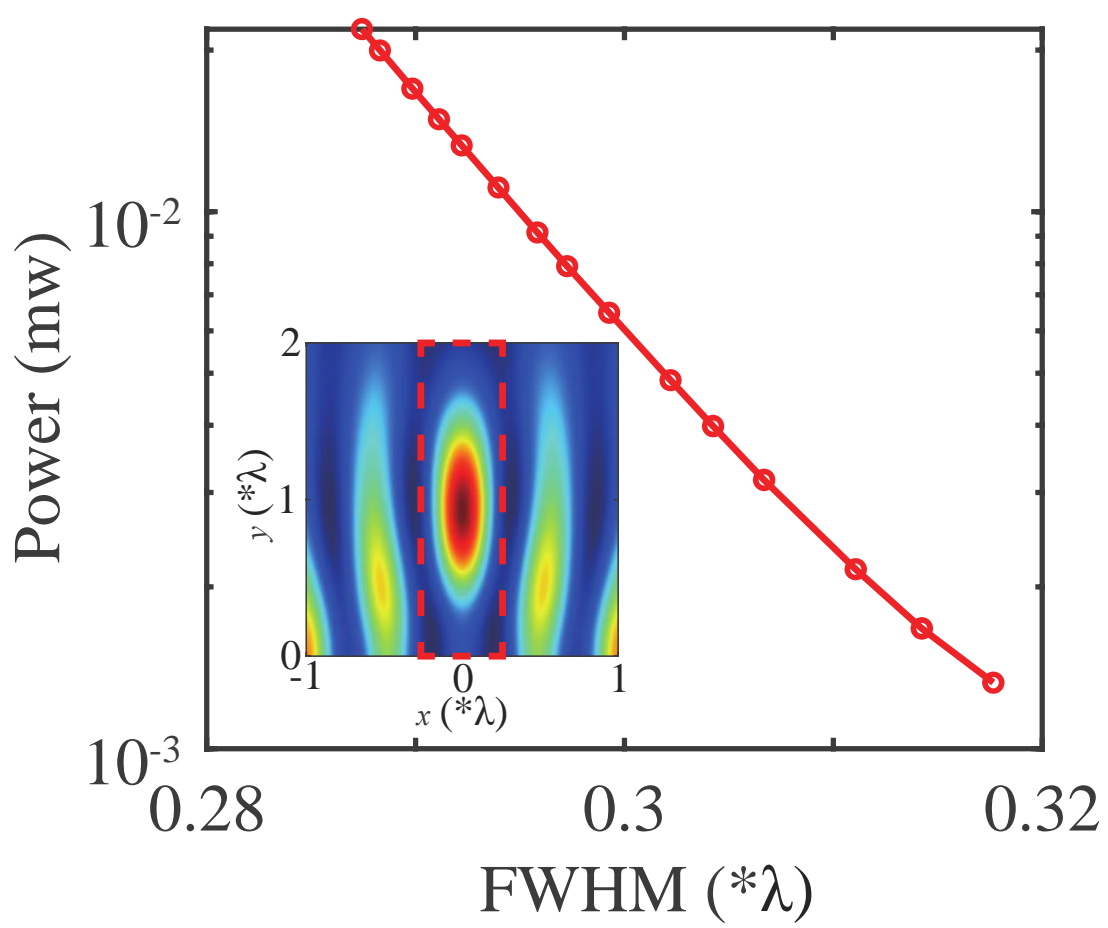

Figure 6. The relationship between the output electric power by PZT layer attached to the plate covering the focusing spot (marked as the red dotted line in the inset) as a function of the measured FWHM. The continuous line is a guide for the eyes.

\section{Sub-wavelength imaging}

Similar to the plane wave focusing, we can also achieve sub-wavelength imaging with the proposed pillared metasurface. In Fig.7a, we show schematically a point source A located at one side of a metasurface with a distance $F$, and its image A' appearing at the opposite side with a focal length $F$. In another word, the point source A and its image A' are symmetrically designed with respect to the metasurface. The corresponding required phase profile can be derived as

$$
\Phi(x)=2 \times \frac{2 \pi}{\lambda}\left(\sqrt{F^{2}+x^{2}}-F\right)+\Phi(x=0)
$$

We also adopt $\Phi(x=0)=-\pi$ for the central pillar corresponding to the strongest resonant status, and the focal length $F$ is chosen as $F=\lambda=423 \mu \mathrm{m}$. The employed material and geometric parameters except pillar's height are the same as in Fig.3. The transmitted intensity field of the imaging effect by the metasurface with 21 pillars is shown as the inset in Fig. $7 b$. The measured focal length is $1.06 \lambda$ (corresponding to $F / D=0.14$ ) and the FWHM reaches a subwavelength value of $0.44 \lambda$. Then we fix the designed focal length at one wavelength and make a sweep in the metasurface's length $D$ (number of pillars). The behavior of FWHM as a function of $F / D$ is shown 
as the dotted curve in Fig. 7b. Similar to Fig.3, the measured focal length is used in the ratio $F / D$. From Fig.7b, one can observe that the FWHM of the imaging spot can also achieve values below $0.5 \lambda$ with the same physical mechanism as in Fig.3, showing a quasi linear relationship with $F / D$. It is found that when the metasurface consists of 13 or more pillars, the focal length of the image always keeps stable as $1.06 \lambda$. In Fig. $7 \mathrm{~b}$, the highest point with $F / D=0.23$ and FWHM=0.46 $\lambda$ corresponds to the number of pillars equal to 13 .

(a)

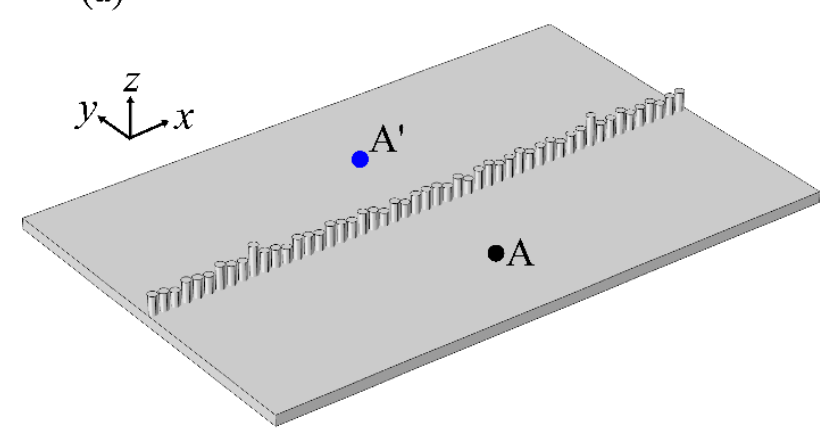

(b)

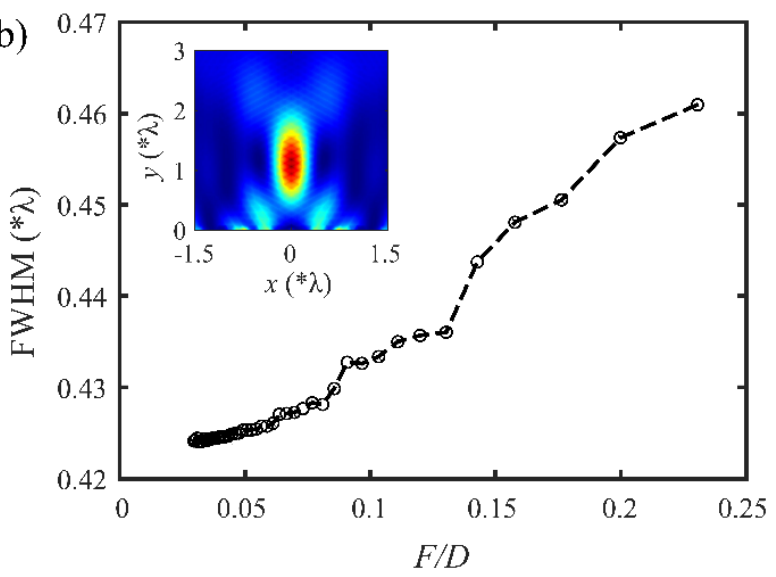

Figure 7. (a) Illustration of imaging effect with the pillared metasurface where $A$ is the point source and $\mathrm{A}^{\prime}$ is the imaging spot. (b) The relation between $F / D$ and FWHM when sweeping the number of pillars in the metasurface while keeping the other parameters fixed. The inset shows the intensity field of imaging effect when the object is at a distance $\lambda$ of the metasurface and the number of pillars is 21 .

\section{Summary}

In summary, we proposed a new type of pillared elastic metasurface consisting of a line of gradient resonant pillars with subwavelength diameter on a homogeneous plate, which is able to provide full phase range manipulation and high transmissions. Subwavelength focusing is achieved by designing gradient pillared metasurface and the results are validated by the Green's function-based Huygens-Fresnel principle (analytical) and the finite element method (numerical) approaches. We also show linear relation between $F / D$ and FWHM in the subwavelength focusing effect. There is a wide range of parameters to keep FWHM smaller than half a wavelength as long as $F / D$ remains within a certain limit with the underlying physical mechanism being the interference and diffraction of the gradient sub-sources in the near field. It is also found that the minima of FWHM by the two approaches can be predicted from the data fitting. We further demonstrated an excellent robustness to maintain the subwavelength behaviors against disorder in 
the geometrical parameters or in the frequency shifts of the incident wave that may exist in real sample fabrication and experimental measurement of the focal length and the FWHM. Since the surface's curvatures will be also enhanced when FWHM is smaller, the pillared metasurfaces bring significant impact on the increase of the energy density for harvesting application, namely promoting the output electric power by more than one order of magnitude. We finally demonstrated subwavelength imaging effect with FWHM smaller than half a wavelength by the proposed metasurface. Since the rigidity of the homogenous plate is conserved, the proposed metasurfaces bring a new way for subwavelength wave manipulation that can be useful for several applications such as energy harvesting, sensing, wave communication or nondestructive evaluation.

\section{Acknowledgment}

This work was supported by the National Natural Science Foundation of China (Grant No. 11902223), the Shanghai Pujiang Program (Grant No. 19PJ1410100), the program for professor of special appointment (Eastern Scholar) at Shanghai Institutions of Higher Learning, the Fundamental Research Funds for the Central Universities, and the High-Level Foreign expert Program.

\section{Reference}

[1] N. Yu, F. Capasso, Flat optics with designer metasurfaces, Nature materials, 13 (2014) 139-150.

[2] F. Ding, A. Pors, S.I. Bozhevolnyi, Gradient metasurfaces: a review of fundamentals and applications, Reports on Progress in Physics, 81 (2017) 026401.

[3] A.V. Kildishev, A. Boltasseva, V.M. Shalaev, Planar photonics with metasurfaces, Science, 339 (2013) 1232009.

[4] N. Yu, P. Genevet, M.A. Kats, F. Aieta, J.-P. Tetienne, F. Capasso, Z. Gaburro, Light propagation with phase discontinuities: generalized laws of reflection and refraction, science, 334 (2011) 333-337.

[5] D. Lin, P. Fan, E. Hasman, M.L. Brongersma, Dielectric gradient metasurface optical elements, science, 345 (2014) 298-302.

[6] M. Khorasaninejad, W.T. Chen, R.C. Devlin, J. Oh, A.Y. Zhu, F. Capasso, Metalenses at visible wavelengths: Diffraction-limited focusing and subwavelength resolution imaging, Science, 352 (2016) 1190-1194.

[7] Y. Xie, W. Wang, H. Chen, A. Konneker, B.-I. Popa, S.A. Cummer, Wavefront modulation and subwavelength diffractive acoustics with an acoustic metasurface, Nature communications, 5 (2014) 5553.

[8] Y. Li, B. Liang, Z.-m. Gu, X.-y. Zou, J.-c. Cheng, Reflected wavefront manipulation based on ultrathin planar acoustic metasurfaces, Scientific reports, 3 (2013) 2546.

[9] K. Tang, C. Qiu, M. Ke, J. Lu, Y. Ye, Z. Liu, Anomalous refraction of airborne sound through ultrathin metasurfaces, Scientific reports, 4 (2014) 6517.

[10] Y. Jin, R. Kumar, O. Poncelet, O. Mondain-Monval, T. Brunet, Flat acoustics with soft gradient-index 
metasurfaces, Nature communications, 10 (2019) 143.

[11] M. Amin, O. Siddiqui, W. Orfali, M. Farhat, A. Khelif, Resonant beam steering and carpet cloaking using an acoustic transformational metascreen, Physical Review Applied, 10 (2018) 064030.

[12] H. Zhu, F. Semperlotti, Anomalous refraction of acoustic guided waves in solids with geometrically tapered metasurfaces, Physical Review Letters, 117 (2016) 034302.

[13] Y. Liu, Z. Liang, F. Liu, O. Diba, A. Lamb, J. Li, Source illusion devices for flexural Lamb waves using elastic metasurfaces, Physical Review Letters, 119 (2017) 034301.

[14] Y. Jin, B. Bonello, R.P. Moiseyenko, Y. Pennec, O. Boyko, B. Djafari-Rouhani, Pillar-type acoustic metasurface, Physical Review B, 96 (2017) 104311.

[15] H. Lee, J.K. Lee, H.M. Seung, Y.Y. Kim, Mass-Stiffness substructuring of an elastic metasurface for full transmission beam steering, Journal of the Mechanics and Physics of Solids, 112 (2018) 577-593.

[16] L. Cao, Z. Yang, Y. Xu, B. Assouar, Deflecting flexural wave with high transmission by using pillared elastic metasurface, Smart Materials and Structures, 27 (2018) 075051.

[17] Y. Chen, X. Li, H. Nassar, G. Hu, G. Huang, A programmable metasurface for real time control of broadband elastic rays, Smart Materials and Structures, 27 (2018) 115011.

[18] L. Cao, Z. Yang, Y. Xu, Z. Chen, Y. Zhu, S.-W. Fan, K. Donda, B. Vincent, B. Assouar, Pillared elastic metasurface with constructive interference for flexural wave manipulation, Mechanical Systems and Signal Processing, 146 (2021) 107035 .

[19] W. Wang, Y. Jin, W. Wang, B. Bonello, B. Djafari-Rouhani, R. Fleury, Robust Fano resonance in a topological mechanical beam, Physical Review B, 101 (2020) 024101.

[20] Y. Jin, W. Wang, B. Djafari-Rouhani, Asymmetric topological state in an elastic beam based on symmetry principle, International Journal of Mechanical Sciences, 186 (2020) 105897.

[21] P.A. Deymier, Acoustic metamaterials and phononic crystals, Springer Science \& Business Media2013.

[22] S.A. Cummer, J. Christensen, A. Alù, Controlling sound with acoustic metamaterials, Nature Reviews Materials, 1 (2016) 16001.

[23] G. Ma, P. Sheng, Acoustic metamaterials: From local resonances to broad horizons, Science advances, 2 (2016) e1501595.

[24] Y. Jin, B. Djafari-Rouhani, D. Torrent, Gradient index phononic crystals and metamaterials, Nanophotonics, 8 (2019) 685-701.

[25] Y. Jin, W. Wang, Z. Wen, D. Torrent, B. Djafari-Rouhani, Topological states in twisted pillared phononic plates, Extreme Mechanics Letters, 39 (2020) 100777.

[26] M. Addouche, M.A. Al-Lethawe, A. Choujaa, A. Khelif, Superlensing effect for surface acoustic waves in a pillarbased phononic crystal with negative refractive index, Applied Physics Letters, 105 (2014) 023501.

[27] M.A. Al-Lethawe, M. Addouche, A. Khelif, S. Guenneau, All-angle negative refraction for surface acoustic waves in pillar-based two-dimensional phononic structures, New Journal of Physics, 14 (2012) 123030.

[28] M. Rupin, S. Catheline, P. Roux, Super-resolution experiments on lamb waves using a single emitter, Applied Physics Letters, 106 (2015) 024103.

[29] A. Sukhovich, B. Merheb, K. Muralidharan, J. Vasseur, Y. Pennec, P.A. Deymier, J. Page, Experimental and theoretical evidence for subwavelength imaging in phononic crystals, Physical review letters, 102 (2009) 154301.

[30] N. Kaina, F. Lemoult, M. Fink, G. Lerosey, Negative refractive index and acoustic superlens from multiple scattering in single negative metamaterials, Nature, 525 (2015) 77.

[31] Y. Zhu, L. Cao, A. Merkel, S.-W. Fan, B. Assouar, Bifunctional superlens for simultaneous flexural and acoustic wave superfocusing, Applied Physics Letters, 116 (2020) 253502.

[32] Y.-X. Shen, Y.-G. Peng, F. Cai, K. Huang, D.-G. Zhao, C.-W. Qiu, H. Zheng, X.-F. Zhu, Ultrasonic super- 
417 oscillation wave-packets with an acoustic meta-lens, Nature Communications, 10 (2019) 3411.

418 [33] A.A. Maznev, O.B. Wright, Upholding the diffraction limit in the focusing of light and sound, Wave Motion, 68 419 (2017) 182-189.

420 [34] G. Lerosey, J. De Rosny, A. Tourin, M. Fink, Focusing beyond the diffraction limit with far-field time reversal, 421 Science, 315 (2007) 1120-1122.

422 [35] M. Dubois, E. Bossy, S. Enoch, S. Guenneau, G. Lerosey, P. Sebbah, Time-driven superoscillations with negative 423 refraction, Physical Review Letters, 114 (2015) 013902.

424 [36] T. Liu, F. Chen, S. Liang, H. Gao, J. Zhu, Subwavelength Sound Focusing and Imaging Via Gradient Metasurface425 Enabled Spoof Surface Acoustic Wave Modulation, Physical Review Applied, 11 (2019) 034061.

426 [37] C. Ma, S. Kim, N.X. Fang, Far-field acoustic subwavelength imaging and edge detection based on spatial filtering 427 and wave vector conversion, Nature communications, 10 (2019) 204.

428 [38] G. Ma, X. Fan, F. Ma, J. de Rosny, P. Sheng, M. Fink, Towards anti-causal Green's function for three-dimensional 429 sub-diffraction focusing, Nature Physics, 14 (2018) 608.

430 [39] L. Chen, W. Wang, G. Nie, Y. Jin, D. Torrent, B. Djafari-Rouhani, Broadband asymmetric propagation in pillared 431 meta-plates, Crystals, 10 (2020) 702.

432 [40] D. Torrent, D. Mayou, J. Sánchez-Dehesa, Elastic analog of graphene: Dirac cones and edge states for flexural waves in thin plates, Physical Review B, 87 (2013) 115143.

434 [41] X. Jiang, B. Liang, J. Yang, J. Yang, J.-c. Cheng, Acoustic planar antireflective focusing lens with sub-diffraction435 limit resolution based on metamaterials, Journal of Applied Physics, 123 (2018) 091717.

436 [42] X. Jiang, Y. Li, D. Ta, W. Wang, Ultrasonic sharp autofocusing with acoustic metasurface, Physical Review B, $437102(2020) 064308$.

438 [43] M. Oudich, Y. Li, Tunable sub-wavelength acoustic energy harvesting with a metamaterial plate, Journal of 439 Physics D: Applied Physics, 50 (2017) 315104. 